\title{
Contrast Enhancing Colour to Grey
}

\author{
Ali Alsam \\ Sør-Trøndelag University College, Trondheim, \\ Norway
}

\begin{abstract}
A spatial algorithm to convert colour images to greyscale is presented. The method is very fast and results in increased local and global contrast. At each image pixel, three weights are calculated. These are defined as the difference between the blurred luminance image and the colour channels: red, green and blue. The higher the difference the more weight is given to that channel in the conversion. The method is multi-resolution and allows the user to enhance contrast at different scales. Results based on three colour images show that the method results in higher contrast than luminance and two spatial methods: Socolinsky and Wolff 12 and Alsam and Drew 3].
\end{abstract}

\section{Introduction}

Colour images contain information about the intensity, hue and saturation of the physical scenes that they represent. From this perspective, the conversion of colour images to black and white has long been defined as: The operation that maps RGB colour triplets to a space which represents the luminance in a colour-independent spatial direction. As a second step, the hue and saturation information are discarded, resulting in a single channel which contains the luminance information.

In the colour science literature, there are, however, many standard colour spaces that serve to separate luminance information from hue and saturation. Standard examples include: CIELab, HSV, LHS, YIQ etc. But the luminance obtained from each of these colour spaces is different.

Assuming the existence of a colour space that separates luminance information perfectly, we obtain a greyscale image that preserves the luminance information of the scene. Since this information has real physical meaning related to the intensity of the light signals reflected from the various surfaces, we can redefine the task of converting from colour to black and white as: An operation that aims at preserving the luminance of the scene.

In recent years, research in image processing has moved away from the idea of preserving the luminance of a single image pixel to methods that include spatial context, thus including simultaneous contrast effects. Including the spatial context means that we need to generate the intensity of an image pixel based on its neighbourhood. Further, for certain applications, preserving the luminance information per se might not result in the desired output. As an example, an equi-luminous image may easily have pixels with very different hue and saturation. However, equating grey with luminance results in a flat uniform grey. So we wish to retain colour regions while best preserving achromatic information.

A.-B. Salberg, J.Y. Hardeberg, and R. Jenssen (Eds.): SCIA 2009, LNCS 5575, pp. 588 596, 2009.

(C) Springer-Verlag Berlin Heidelberg 2009 
To proceed, we state that a more encompassing definition of colour to greyscale conversion is: An operation that reduces the number of channels from three to one while preserving certain, user defined, image attributes. As an example, Bala and Eschbach [4, introduced an algorithm to convert colour images to greyscale while preserving colour edges. Socolinsky and Wolff [1]2, developed a technique for multichannel image fusion with the aim of preserving contrast. More recently, Alsam and Drew [3] introduced the idea of defining contrast as the maximum change in any colour channel along the $x$ and $y$ directions. In general, we can state that the literature of spatial colour to grey is based on the idea of preserving the differences between colour and grey regions in the original image.

In this paper, a new approach to the problem of converting colour images to grey is taken. The approach is based on the photographic definition of what is an optimal, or beautiful, black and white image. During the preparation work for this article, I surveyed the views of many professional photographers. Their response was exclusively that a black and white image is aesthetically more beautiful than the colour original because it has higher global and local contrast. This view is supported in the vision science literature [5] where: It is well known that contrast between black and white is greater than that between red-green or blue-yellow. Based on this, in this paper, an optimal conversion from colour to black and white image is defined as an algorithm that converts colour values to grey while maximizing the local contrast. A new definition of contrast is presented and the conversion is performed to optimize it.

\section{Background}

As stated in the introduction, the best transformation from a multi-channel image to greyscale depends on the given definition. It is possible, however, to divide the solution domain into two groups. In the first, we have global projection based methods. In the second, we have spatial methods.

Global methods can further be divided into image independent and image dependent algorithms. Image independent algorithms, such as the calculation of luminance, assume that the transformation from colour to grey is related to the cone sensitives of the human eye. Based on that, the luminance approach is defined as a weighted sum of the red, green and blue values of the image without any measure of the image content. Further, the weights assigned to the red, green and blue channels are derived from vision studies where it is known that the eye is more sensitive to green than to red and blue.

To improve upon the performance of the image-independent averaging methods, we can incorporate statistical information about the image's colour, or multi-spectral, information. Principal component analysis (PCA) achieves this by considering the colour information as vectors in an $n$-dimensional space. The covariance matrix of all the colour values in the image, is analyzed using PCA and the principal vector with the largest principal value is used to project the image data onto the vector's one dimensional space [6. Generally speaking, using PCA, more weight is given to channels with more intensity. It has, however, been shown that PCA shares a common problem with the global averaging techniques [2]: The contrast between adjacent pixels in the grey reproduction is always less 
than the original. This problem becomes more noticeable when the number of channels increases [2].

Spatial methods are based on the assumption that the transformation from colour to greyscale needs to be defined such that differences between pixels are preserved. Bala and Eschbach [4, introduced a two step algorithm. In the first step the luminance image is calculated based on a global projection. In the second, the chrominance edges that are not present in the luminance are added to the luminance. Similarly, Grundland and Dodgson [7, introduced an algorithm that starts by transforming the image to $Y I Q$ colour space. The $Y$-channel is assumed to be the luminance of the image and treated separately from the the chrominance $I Q$ plane. Based on the chrominance information in the $I Q$ plane, they calculate a single vector: The predominant chromatic change vector. The final greyscale image is defined as a weighted sum of the luminance $Y$ and the projection of the 2-dimensional $I Q$ onto the predominant vector.

Socolinsky and Wolff [12], developed a technique for multichannel image fusion with the aim of preserving contrast. In their work, these authors use the Di Zenzo structure-tensor matrix [8] to represent contrast in a multiband image. The interesting idea added to [8] was to suggest re-integrating the gradient produced in Di Zenzo's approach into a single, representative, grey channel encapsulating the notion of contrast. Connah et al. 9] compared six algorithms for converting colour images to greyscale. Their findings indicate that the algorithm presented by Socolinsky and Wolff [12] results in visually preferred rendering.

The Di Zenzo matrix allows us to represent contrast at each image pixel by utilising a $2 \times 2$ symmetric matrix whose elements are calculated based on the derivatives of the colour channels in the horizontal and vertical directions. Socolinsky and Wolff defined the maximum absolute colour contrast to be the square root of the maximum eigenvalue of the Di Zenzo matrix along the direction of the associated eigenvector. In [1, Socolinsky and Wolff noted that the key difference between contrast in the greyscale case and that in a multiband image is that, in the latter, there is no preferred orientation along the maximum contrast direction. In other words, contrast is defined along a line, not a vector.

To resolve the resulting sign ambiguity, Alsam and Drew 3 introduced the idea of defining contrast as the maximum change in any colour channel along the $x$ and $y$ directions. Using the maximum change resolves the sign ambiguity and results in a very fast algorithm that was shown to produce better results than those achieved by Socolinsky and Wolff [12].

\section{Contrast Enhancing}

RGB colour images are commonly converted to greyscale using a weighted sum of the form:

$$
G r(x, y)=\alpha R(x, y)+\beta G(x, y)+\gamma B(x, y)
$$

where $\alpha, \beta$ and $\gamma$ are positive scalars that sum to one.

At the very heart of the algorithm presented in this article is the question: Which local weights $\alpha(x, y), \beta(x, y)$ and $\gamma(x, y)$ would result in maximizing the contrast of the greyscale image pixel $\operatorname{Gr}(\mathrm{x}, \mathrm{y})$ ? To answer this question we need to first define contrast. 
In the image processing literature, contrast, for a single channel, is define as the deviation from the mean of an $n \times n$ neighborhood. As an example the contrast at the red pixel $R(x, y)$ is:

$$
C_{r}(x, y)=R(x, y)-\sum_{i=1}^{n} \sum_{j=1}^{n} \lambda(i, j) R(i, j)
$$

where $\lambda(i, j)$ are the weights assigned to each image pixel. We note that contrast as defined in (2) represents the high frequency elements of the red channel.

The main contribution of this paper is to define contrast enhancing weights based on the original colour image and a greyscale version calculated as a weighted sum. The author's argument is as follows: The greyscale scale image defined in Equation (1), is a weighted average of all three colour values, red, green and blue at pixel $(x, y)$. To arrive at a similar formulation as in Equation (2), we calculate the difference between red, green and blue at pixel $(x, y)$ and the average of an $n \times n$ neighborhood calculated based on the greyscale image $G r$, i.e.:

$$
\begin{aligned}
& C_{r g}(x, y)=\left|R(x, y)-\sum_{i=1}^{n} \sum_{j=1}^{n} \lambda(i, j) G r(i, j)\right|+\kappa \\
& C_{g g}(x, y)=\left|G(x, y)-\sum_{i=1}^{n} \sum_{j=1}^{n} \lambda(i, j) G r(i, j)\right|+\kappa \\
& C_{b g}(x, y)=\left|B(x, y)-\sum_{i=1}^{n} \sum_{j=1}^{n} \lambda(i, j) G r(i, j)\right|+\kappa
\end{aligned}
$$

where $\kappa$ is a small positive scalar used to avoid division with zero. The scalar $\kappa$ can also be used as a regularization factor where to larger the value the more the closer the resultant weights $C_{r g}(x, y), C_{g g}(x, y)$ and $C_{b g}(x, y)$ are to each other. The weights, $C_{r g}(x, y), C_{g g}(x, y)$ and $C_{b g}(x, y)$ represent the level of high frequency, based on the individual channels, lost when converting an RGB colour image to grey. Thus, if we use those weights to convert the colour image to black and white we get a greyscale representation that gives more weight to the channel that loses most information in the conversion. In other words: The greyscale value $\operatorname{Gr}(x, y)$ is the average of the three channels and the weights $C_{r g}(x, y), C_{g g}(x, y)$ and $C_{b g}(x, y)$ are the spatial difference from the average. Using those would, thus, increase the contrast of $\operatorname{Gr}(x, y)$. The formulation given in Equations (3), (4), (5), however, suffers from a main drawback: For a flat region, one with a single colour, the weights $, C_{r g}(x, y), C_{g g}(x, y)$ and $C_{b g}(x, y)$ will not have a spatial meaning. Said differently, contrast at a single pixel or a region with no colour change is not defined. To resolve this problem we modify the weights $C_{r g}(x, y), C_{g g}(x, y)$ and $C_{b g}(x, y)$ :

$$
C_{R g}(x, y)=\left|D(x, y) \times\left(R(x, y)-\sum_{i=1}^{n} \sum_{j=1}^{n} \lambda(i, j) G r(i, j)\right)\right|+\kappa
$$




$$
\begin{aligned}
& C_{G g}(x, y)=\left|D(x, y) \times\left(G(x, y)-\sum_{i=1}^{n} \sum_{j=1}^{n} \lambda(i, j) G r(i, j)\right)\right|+\kappa \\
& C_{B g}(x, y)=\left|D(x, y) \times\left(B(x, y)-\sum_{i=1}^{n} \sum_{j=1}^{n} \lambda(i, j) G r(i, j)\right)\right|+\kappa
\end{aligned}
$$

where the spatial weights $D(x, y)$ are defined as:

$$
\begin{aligned}
D(x, y)= & \left\|R(x, y)-\sum_{i=1}^{n} \sum_{j=1}^{n} \lambda(i, j) R(i, j)\right\| \\
& +\left\|G(x, y)-\sum_{i=1}^{n} \sum_{j=1}^{n} \lambda(i, j) G(i, j)\right\| \\
& +\left\|B(x, y)-\sum_{i=1}^{n} \sum_{j=1}^{n} \lambda(i, j) B(i, j)\right\|
\end{aligned}
$$

Introducing the difference $D(x, y)$ into the calculation of the weights $C_{R g}(x, y)$, $C_{G g}(x, y)$ and $C_{B g}(x, y)$ means that contrast is only enhanced at regions with colour transition.

Finally, based on $C_{R g}(x, y), C_{G g}(x, y)$ and $C_{B g}(x, y)$ we define the weights: $\alpha(x, y), \beta(x, y)$ and $\gamma(x, y)$ as:

$$
\begin{aligned}
& \alpha(x, y)=\frac{C_{R g}(x, y)}{C_{R g}(x, y)+C_{G g}(x, y)+C_{B g}(x, y)} \\
& \gamma(x, y)=\frac{C_{G g}(x, y)}{C_{R g}(x, y)+C_{G g}(x, y)+C_{B g}(x, y)} \\
& \beta(x, y)=\frac{C_{B g}(x, y)}{C_{R g}(x, y)+C_{G g}(x, y)+C_{B g}(x, y)}
\end{aligned}
$$

For completeness, we modify the conversion given in Equation (1) from colour to grey:

$$
G r(x, y)=\alpha(x, y) R(x, y)+\beta(x, y) G(x, y)+\gamma(x, y) B(x, y)
$$

\section{Experiments}

Figure 1, London photo, shows a colour image with the luminance rendering to its right. In the second, third, fourth and fifth rows the difference maps defined in Equation (9) are shown in the first column and the results achieved with the present method in the second. These results are achieved by blurring the luminance image by: $5 \times 5,10 \times 10,15 \times 15$ and $25 \times 25$ Gaussian kernels respectively. As seen, the contrast increases with the increasing size of the kernel.

In Figure 2, two women, the same layout as in Figure 1 is used. Again, we notice that the contrast increases with the increasing size of the kernel. We note, however, that finer details are better preserved at lower scales. This suggests that the method can be used to combine results at different scales. The best way to combine different scales is, however, left as future work.

In Figure 3, daughter and father, the colour original is shown at the top left corner and the luminance rendition is shown at the top right corner. In the 

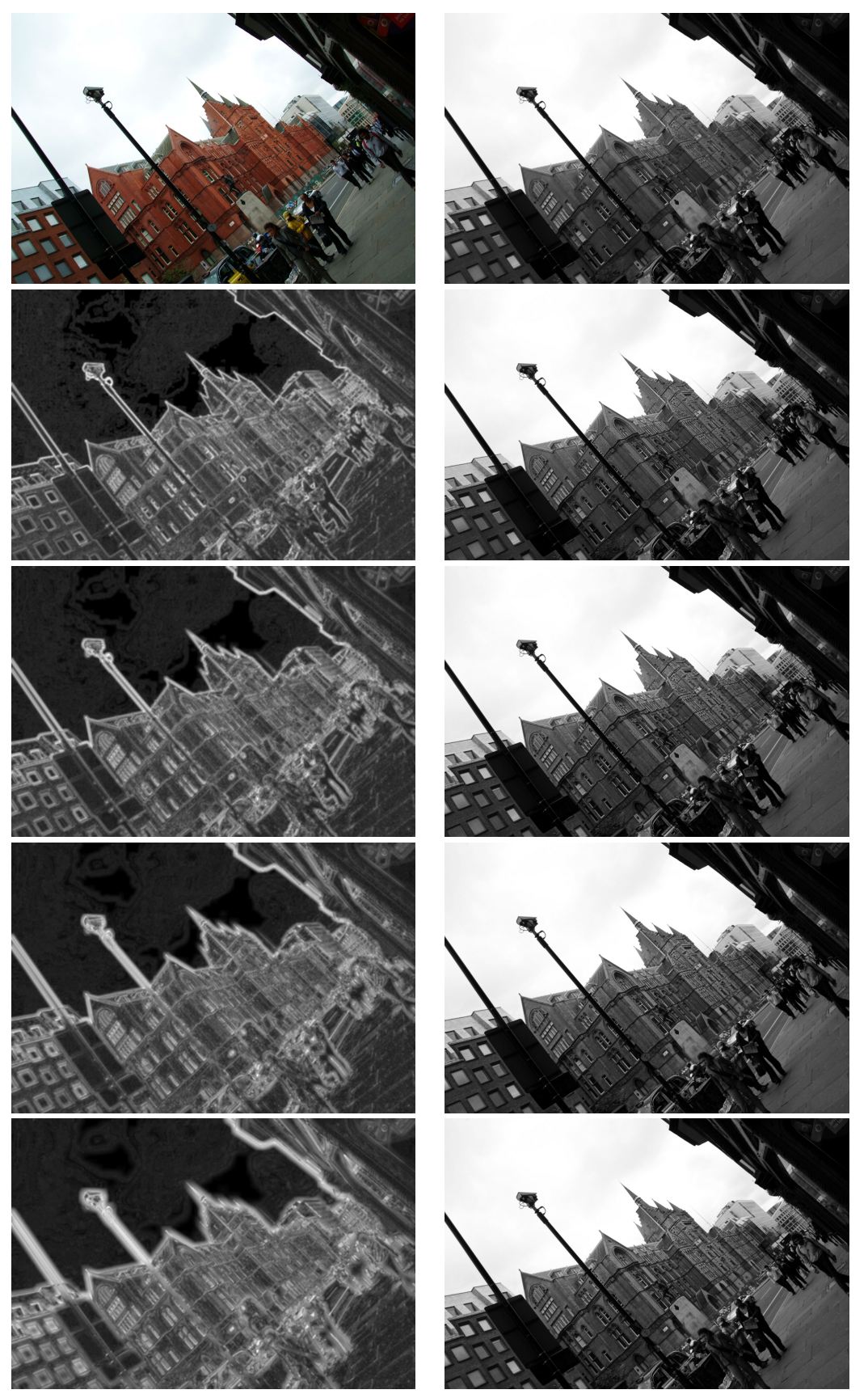

Fig. 1. London photo: top row a colour image with the luminance rendering to its right. In the second, third, fourth and fifth rows the difference maps defined in Equation (9) are shown in the first column and the results achieved with the present method in the second. These results are achieved by blurring the luminance image by: $5 \times 5,10 \times 10$, $15 \times 15$ and $25 \times 25$ Gaussian kernels respectively. 

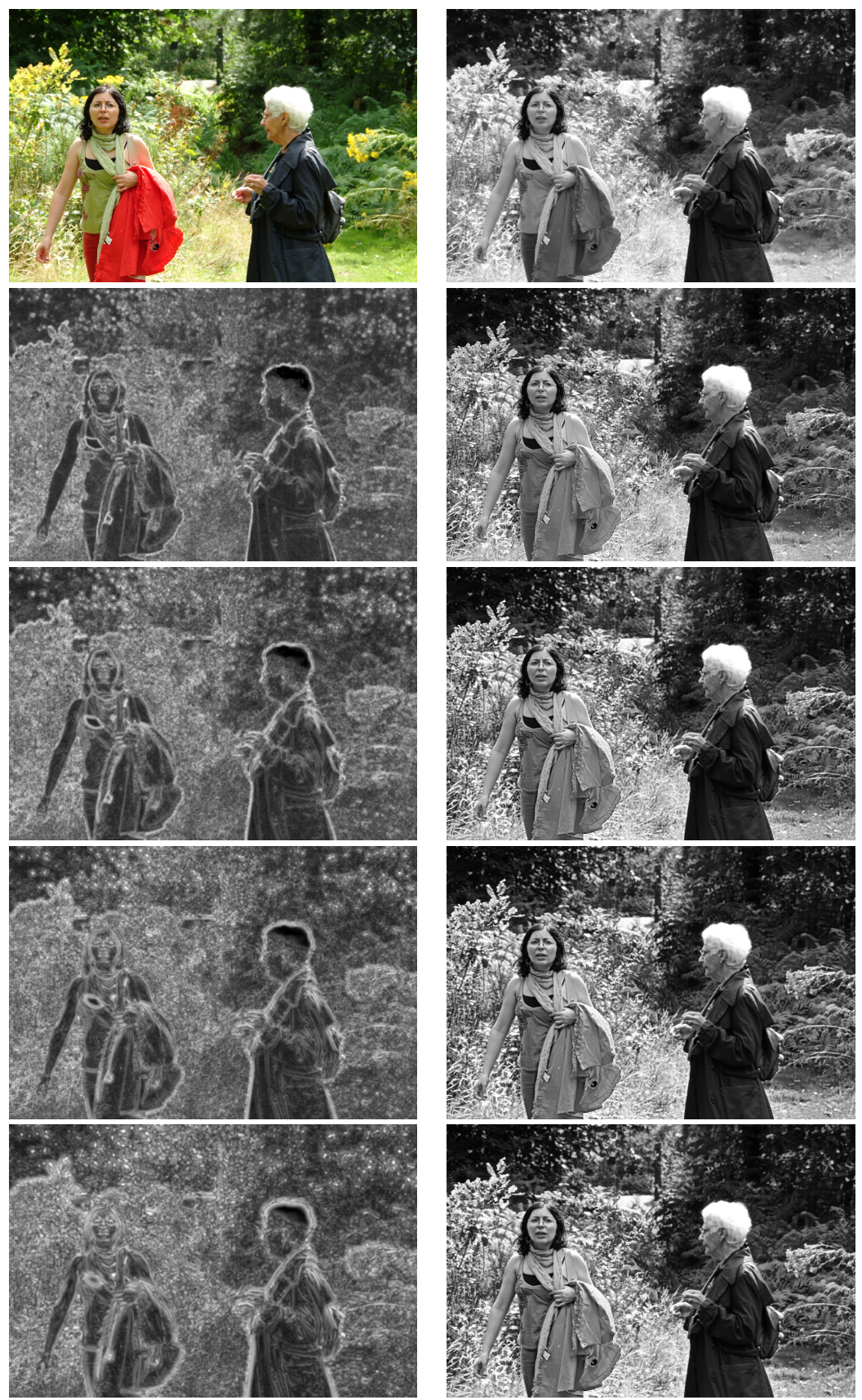

Fig. 2. Two women: top row a colour image with the luminance rendering to its right. In the second, third, fourth and fifth rows the difference maps defined in Equation (9) are shown in the first column and the results achieved with the present method in the second. These results are achieved by blurring the luminance image by: $5 \times 5,10 \times 10$, $15 \times 15$ and $25 \times 25$ Gaussian kernels respectively. 

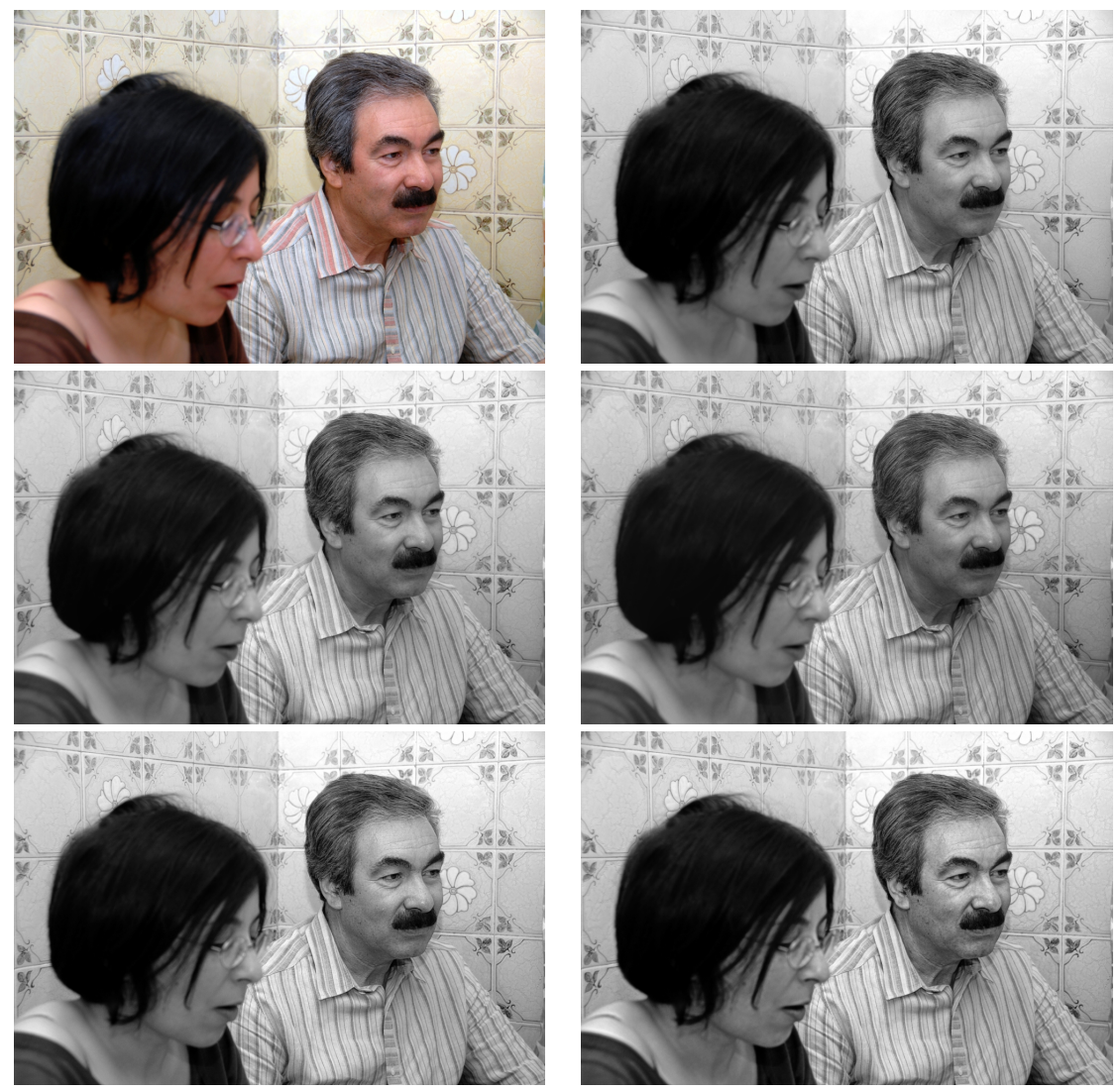

Fig. 3. Daughter and father: top row a colour image with the luminance rendering to its right. In the second row, the results obtained by Socolinsky and Wolff are shown in the first column and those achieved by Alsam and Drew are shown in the second column. The results obtained with the present method based on a $5 \times 5$ and $15 \times 15$ Gaussian kernels are shown in the first and second columns, the third row, respectively.

second row, the results obtained by Socolinsky and Wolff $[12$ are shown to the left and those achieved by Alsam and Drew [3] to the right. In the third row the present method is shown with a blurring of $5 \times 5$ to the left and $15 \times 15$ to the right. We note that the present method achieves the highest contrast out of all other methods.

\section{Conclusions}

Starting with the idea that a black and white image can be optimized to have higher contrast than the colour original, a spatial contrast-enhancing algorithm to convert colour images to greyscale was presented. At each image pixel, three spatial weights are calculated. These are derived to increase the difference between the resulting greyscale value and the mean of the luminance at the given 
image pixel. Results based on general photographs show that the method results in visually preferred rendering. Given that contrast is defined at different spatial scales, the method can be used to combine contrast in a pyramidal fashion.

\section{References}

1. Socolinsky, D.A., Wolff, L.B.: A new visualization paradigm for multispectral imagery and data fusion. In: CVPR, pp. I:319-324 (1999)

2. Socolinsky, D.A., Wolff, L.B.: Multispectral image visualization through first-order fusion. IEEE Trans. Im. Proc. 11, 923-931 (2002)

3. Alsam, A., Drew, M.S.: Fastcolour2grey. In: 16th Color Imaging Conference: Color, Science, Systems and Applications, Society for Imaging Science \& Technology (IS\&T)/Society for Information Display (SID) joint conference, Portland, Oregon, pp. 342-346 (2008)

4. Bala, R., Eschbach, R.: Spatial color-to-grayscale transform preserving chrominance edge information. In: 14th Color Imaging Conference: Color, Science, Systems and Applications, pp. 82-86 (2004)

5. Hunt, R.W.G.: The Reproduction of Colour, 5th edn. Fountain Press, England (1995)

6. Lillesand, T.M., Kiefer, R.W.: Remote Sensing and Image Interpretation, 2nd edn. Wiley, New York (1994)

7. Grundland, M., Dodgson, N.A.: Decolorize: Fast, contrast enhancing, color to grayscale conversion. Pattern Recognition 40(11), 2891-2896 (2007)

8. Di Zenzo, S.: A note on the gradient of a multi-image. Comp. Vision, Graphics, and Image Proc. 33, 116-125 (1986)

9. Connah, D., Finlayson, G.D., Bloj, M.: Seeing beyond luminance: A psychophysical comparison of techniques for converting colour images to greyscale. In: 15th Color Imaging Conference: Color, Science, Systems and Applications, pp. 336-341 (2007) 